\title{
EFEITOS DO ÁCIDO ASCÓRBICO NOS BIOMARCADORES DE ESTRESSE OXIDATIVO EM NADADORES DE ELITE
}

\author{
EFFECTS OF ASCORBIC ACID ON OXIDATIVE STRESS BIOMARKERS OF ELITE SWIMMERS
}

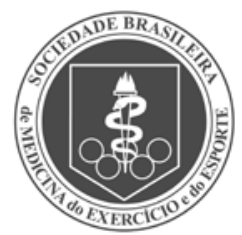

Artigo Original

\section{EFECTOS DEL ÁCIDO ASCÓRBICO EN LOS BIOMARCADORES DE ESTRÉS OXIDATIVO} EN NADADORES DE ELITE

Monike Garlipp-Picchi

(Nutricionista)

Rafael Deminice' (Educador Físico)

Paula Paião Ovídio' (Biomédica)

Alceu Afonso Jordão' (Biólogo)

1. Laboratório de Nutrição e

Metabolismo, Faculdade de

Medicina de Ribeirão Preto da

Universidade de São Paulo -

Ribeirão Preto, SP, Brasil.

\section{Correspondência:}

Laboratório de Nutrição e

Metabolismo. Faculdade de

Medicina de Ribeirão Preto da

Universidade de São Paulo.

Av. Bandeirantes, 3.900, 14049-900

Ribeirão Preto, SP, Brasil

monike.garlipp@usp.br

\section{RESUMO}

Objetivo: O objetivo do estudo foi verificar os efeitos da oferta de vitamina $C$ através de um suplemento alimentar e dieta rica em ácido ascórbico (AA) no estresse oxidativo induzido pelo exercício. Métodos: A amostra foi composta por 13 nadadores de elite (seis homens e sete mulheres) com idades entre 18 e 26 anos. Os mesmos atletas foram submetidos a sessão de exercício agudo em três fases, com diferentes tratamentos: controle (C), dieta rica em AA (D) e suplemento de AA (S), nas quais amostras de sangue foram colhidas antes, imediatamente após e 24 horas depois do exercício. Para comparação entre as fases e etapas foi utilizado o modelo de regressão linear com efeitos mistos. Resultados: O consumo alimentar habitual de antioxidantes não foi diferente entre as fases, apenas a ingestão de AA foi maior na fase $\mathrm{D}$ e $\mathrm{S}$ em relação à fase $\mathrm{C}$. $\mathrm{O}$ uso de dieta rica em AA favoreceu uma menor peroxidação lipídica, devido aos menores valores de hidroperóxidos lipídicos (FOX), diminuição da peroxidação após exercício, pela diminuição de substâncias reativas ao ácido tiobarbitúrico (TBARS) e aumento dos níveis de vitamina C logo após o exercício. O uso de suplemento de AA também conteve a peroxidação lipídica após exercício e aumentou o poder antioxidante, devido aos maiores níveis de glutationa reduzida (GSH). Sem adição de AA os nadadores tiveram um maior dano hepático, pois apresentaram maiores níveis de transaminase- glutâmico-oxalacética (TGO), menores níveis de antioxidantes (vitamina C e GSH) e aumento do ácido úrico. Conclusão: Assim, as mudanças observadas com a adição de AA à dieta de nadadores sugerem um importante papel deste micronutriente na defesa contra o estresse oxidativo induzido pelo exercício.

Palavras-chave: vitamina C, radical livre, suplementação.

\section{ABSTRACT}

Objective: The objective of the present study was to determine the effects of vitamin Coffered through a dietary supplement and an ascorbic acid (AA)-rich diet on exercise-induced oxidative stress. Methods: The sample consisted of 13 elite swimmers ( 6 men and 7 women) aged 18 to 26 years. The same athletes were submitted to an acute exercise session in 3 phases, with different treatments: control (C), AA-rich diet (D) and AA supplement (S), where blood samples were collected before, immediately after and 24 hours after exercise. A mixed effects linear regression model was used to compare phases and stages. Results: The habitual consumption of antioxidants did not differ between phases, except that AA intake was higher during the D and $S$ phases than during the C phase. The use of an AA-rich diet provided lower lipid peroxidation due to lower lipid hydroperoxide (FOX) values, a reduction of peroxidation after exercise due to reduction of thiobarbituric acid reactive substances (TBARS), and an increase in vitamin Clevels after exercise. The use of an AA supplement also restricted lipid peroxidation after exercise and increased the antioxidant power due to higher levels of reduced glutathione (GSH). Without the addition of AA, the swimmers had greater hepatic damage as shown by higher levels of aspartate aminotransferase (AST), lower antioxidant levels (vitamin ( and GSH) and increased uric acid. Conclusion: Thus, the changes observed after the addition of AA to the diet of swimmers suggest an important role of this micronutrient in the defense against exercise-induced oxidative stress.

Keywords: vitamin C, free radicals, dietary supplementation.

\section{RESUMEN}

Objetivo: El objetivo del estudio fue verificar los efectos del ofrecimiento de vitamina $\mathrm{C}$ mediante un suplemento alimenticio y una dieta rica en ácido ascórbico (AA) para el estrés oxidativo inducido por el ejercicio. Métodos: La muestra se compuso de 13 nadadores de elite (seis hombres y siete mujeres) con edades entre 18 y 26 años. Los mismos atletas fueron sometidos a sesión de ejercicio agudo en tres fases, con tratamientos diferentes: control (C), dieta rica en $A A(D)$ y suplemento de $A A(S)$, en las cuales se recolectaron muestras de sangre antes, inmediatamente después, y 24 horas posteriores al ejercicio. Para comparaciones, entre las fases y etapas, se utilizó el modelo de regresión linear con efectos mixtos. Resultados: El consumo alimentario habitual de antioxidantes no fue diferentes entre las fases, solamente la ingestión de AA fue mayor en las fases D y S, en relación con la fase C. El uso de dieta rica en AA favoreció una menor peroxidación lipídica, debido a los valores menores de hidroperóxidos lipídicos (FOX), disminución de la peroxidación después del ejercicio, por la reducción de sustancias reactivas al ácido tiobarbitúrico (TBARS) y aumento de los niveles de vitamina C inmediatamente después del ejercicio. El uso de suplemento de AA también contuvo la peroxidación lipídica después del ejercicio y aumentó el poder antioxidante, debido a los niveles más altos 
de glutationa reducida (GSH). Sin adición de AA, los nadadores tuvieron un mayor daño hepático, pues presentaron niveles mayores de transaminasa-glutámica-oxalacética (TGO), menores niveles de antioxidantes (vitamina Cy GSH) y aumento del ácido úrico. Conclusión: Así, los cambios observados con la adición de AA a la dieta de nadadores sugieren un importante papel de este micronutriente en la defensa contra el estrés oxidativo inducido por el ejercicio.

Palabras clave: vitamina C, radical libre, suplementación.

Artigo recebido em 09/11/2012, aprovado em 26/09/2013.

\section{INTRODUÇÃO}

A prática regular de exercícios físicos associada a uma dieta balanceada pode ser importante fator na promoção da saúde; entretanto, exercício vigoroso resulta em estresse oxidativo ${ }^{2}$. A frequente realização de exercícios físicos de alta intensidade ou exaustivos pode aumentar a suscetibilidade a lesões, promover a fadiga crônica e overreaching, parcialmente em razão da elevada síntese de espécies reativas de oxigênio (EROs), as quais podem estar envolvidas em diversos processos fisiopatológicos como envelhecimento, câncer, doenças inflamatórias e aterosclerose ${ }^{1,3}$. Por outro lado, as EROs podem ter efeitos considerados positivos para o sistema imune e têm funções metabólicas essenciais para a homeostasia celular'.

A suplementação antioxidante pode ser utilizada nas situações em que os mecanismos normais de defesa do corpo não são suficientes para lidar adequadamente com o aumento da produção de EROs ${ }^{1,4,5}$. Vários estudos que avaliaram o uso de suplementos antioxidantes observaram atenuação de alguns marcadores de estresse oxidativo após exercício ${ }^{5-7}$.

A necessidade de vitaminas e minerais antioxidantes para atletas ainda é a mesma que para indivíduos saudáveis. Porém, se houver uma maior utilização de antioxidantes para conter o estresse oxidativo gerado pelo exercício, as necessidades nutricionais podem ser maiores ${ }^{8}$. Alguns autores supõem que atletas possam apresentar as necessidades relativas a determinados tipos de micronutrientes acima da Recommended Dietary Allowance $e^{9}$, dessa maneira, a suplementação antioxidante seria apropriada ${ }^{10,11}$.

Sendo assim, o objetivo do presente estudo foi comparar os efeitos da oferta de vitamina C através de um suplemento alimentar e de uma dieta rica em ácido ascórbico (AA) nos biomarcadores do estresse oxidativo induzido por exercício físico em nadadores de elite.

\section{MÉTODOS}

\section{Indivíduos}

A amostra foi composta por 13 nadadores de alto rendimento, seis homens e sete mulheres, com idades entre 18 e 26 anos (20,62 $\pm 2,29$ anos). Nenhum indivíduo era tabagista e nenhum fez uso de suplemento antioxidante nas duas semanas antes das coletas de sangue e durante as mesmas. Os atletas eram federados, competidores ranqueados em campeonatos estaduais e nacionais. Para participação na pesquisa, os indivíduos assinaram o Termo de Consentimento Livre e Esclarecido aprovado pelo Comitê de Ética em Pesquisa do Hospital das Clínicas de Ribeirão Preto.

\section{Delineamento do estudo}

A pesquisa consistiu na avaliação dos biomarcadores de estresse oxidativo e antioxidantes em três fases, com intervalo de dois meses entre elas. A primeira denominada fase controle (C), na qual os parâmetros analisados reproduziram os valores basais dos indivíduos. Na segunda fase, fase dieta (D), os indivíduos receberam uma dieta rica em AA por três dias antes das mensurações, através do consumo de um litro de suco de laranja por dia (425,65 mg/d de AA). E a terceira fase, denominada fase suplemento (S), os atletas receberam $500 \mathrm{mg}$ de suplemento de AA três dias antes das análises.

Em cada fase foram feitas coletas de sangue em três etapas: antes do treino (AE), logo após (LA) e 24 horas após o exercício (24 h), como descrito no fluxograma (figura 1). Os participantes fizeram registro alimentar de três dias antes das coletas e do dia da coleta. Compareceram ao laboratório em jejum de 12 horas para coleta de sangue antes do exercício; neste período, foram autorizados a consumir apenas água. Para a realização das dosagens 24 horas após o exercício, os indivíduos foram orientados a não praticar nenhum tipo de exercício físico e fazer novamente jejum de 12 horas.

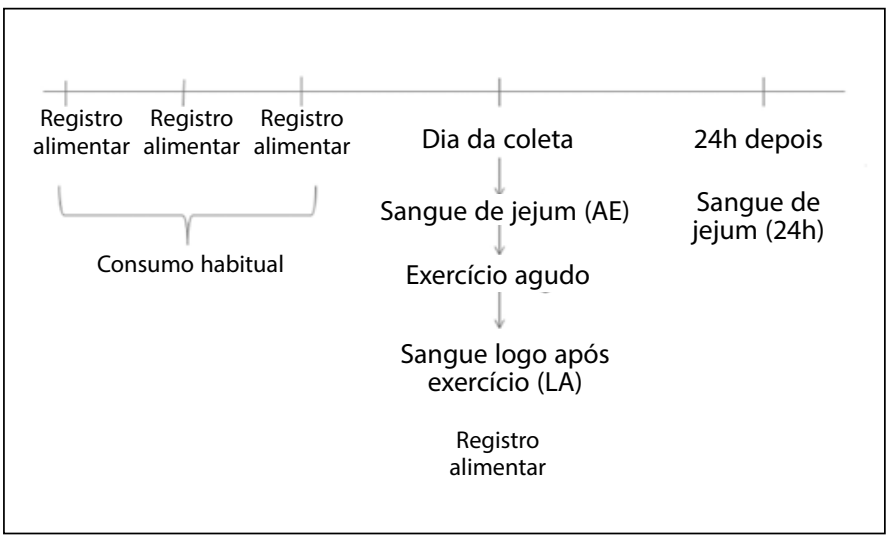

Figura 1. Fluxograma com as etapas da coleta dentro de cada fase do estudo. AE antes do exercício / LA - logo após o exercício / 24 h - 24 horas após o exercício.

Os atletas mantiveram sua rotina de treino durante o período. Entretanto, sabendo que as intensidades são variáveis ao longo dos períodos e para que fosse possível padronizá-la nas diferentes fases, foi combinado com o treinador que fizesse uma padronização do treino na semana das coletas de sangue, permitindo a avaliação dos indivíduos em situação de estresse semelhante.

\section{Avaliação do consumo alimentar}

Para avaliar o consumo alimentar habitual dos participantes foi utilizada a média dos três dias de registro alimentar antes da coleta, que incluía um dia do final de semana. O único nutriente que não representa o consumo habitual é o $A A$, nas fases $D$ e $S$, devido à adição feita pelo estudo. Todos os registros alimentares foram analisados pelo software DietPro 4.0 (Agromídia Software LTDA, Viçosa, MG, 2002). Foram analisados os antioxidantes AA (vitamina C), vitamina E, selênio, zinco e retinol. Tanto o consumo do suco de laranja como o uso de suplementos alimentares foi adicionado às análises. Foram utilizados os valores propostos pelas Dietary Reference Intakes $^{12}$ para avaliar a adequação da ingestão de AA de acordo com os critérios descritos por Cuppari e Schor ${ }^{13}$. 


\section{Avaliação bioquímica}

Foram colhidos, aproximadamente, $4 \mathrm{ml}$ de sangue por punção venosa em tubo a vácuo estéril (Vacutainer - $\mathrm{BD}^{\circledR}{ }^{\circledR}$, contendo ativador de coagulação em cada uma das coletas de sangue. Logo após as coletas, as amostras de sangue foram centrifugadas a 3.500 rotações por minuto (rpm) por 10 minutos à temperatura ambiente. O sobrenadante (soro) foi separado, transferido para um tubo de Eppendorf e armazenado a $-30^{\circ} \mathrm{C}$.

Quanto ao metabolismo, antioxidante e estresse oxidativo foram dosados, biomarcadores de peroxidação lipídica, através da determinação de substâncias reativas ao ácido tiobarbitúrico-TBARS ${ }^{14} \mathrm{e}$ de hidroperóxidos lipídicos pela oxidação do ferro em xilenol laranja (FOX) ${ }^{15}$, glutationa reduzida $(\mathrm{GSH})^{16}$, vitamina $\mathrm{C}^{17}$ e vitamina $\mathrm{E}^{18}$. Foram utilizados kits comerciais Labtest Diagnóstica (Lagoa Santa, MG, Brasil) para dosagem do ácido úrico, da aspartato aminotransferase (AST) e da creatina quinase (CK).

\section{Análise estatística}

As variáveis são apresentadas como média \pm desvio padrão. Para comparação entre as fases e etapas foi utilizado o modelo de regressão linear com efeitos mistos, através do software $S A S^{\circledR} 9.1$, utilizando a PROC MIXED. Para avaliar a força de associação entre as variáveis do estudo foi feito o coeficiente de correlação de Spearman nas correlações por fase e etapas. O nível de significância estatística foi definido em $p<0,05$ em todos os casos.

\section{RESULTADOS}

A maioria dos nadadores $(n=12,92,3 \%)$ referiram fazer uso de suplementos alimentares e $25 \%$ deles $(n=3)$ reportaram uso de suplementos vitamínicos e minerais. O consumo alimentar habitual dos voluntários não foi diferente entre as fases para os antioxidantes vitamina $\mathrm{E}$, alfa-tocoferol, retinol, selênio e zinco. Ainda o consumo alimentar do dia da coleta de sangue foi igual ao consumo habitual. Como esperado, a ingestão habitual de AA foi maior na fase D (242,4\%) e S (343,27\%) em relação à fase C. Os valores de consumo habitual e do dia das coletas estão apresentados na tabela 1.

A maioria dos indivíduos apresentou consumo adequado de AA. Apenas dois indivíduos apresentaram consumo insuficiente na fase $C$ 2. Porém, sem considerar a adição de $A A$ à dieta, $61,5 \%$ dos indivíduos $(n=8)$ apresentariam consumo insuficiente, na fase $D$, se não tivessem feito uso de um litro de suco de laranja; e 38,4\% $(n=5)$, na fase $S$, se não tivessem feito uso de suplemento de $\mathrm{AA}$. O uso adicional deste antioxidante não resultou em consumo acima dos limites máximos recomendados, que variam de 1.800 a $2.000 \mathrm{mg}$ por dia para os atletas do presente estudo.
Os biomarcadores de estresse oxidativo em resposta ao uso adicional de AA e exercício físico estão apresentados nas figuras de 2, 3 e 4. Os resultados mostram que a adição de AA pela dieta e pelo uso de suplemento aumenta significativamente a vitamina C plasmática $(p<0,05)$. Estes resultados demonstram a eficácia das duas diferentes maneiras para consumir vitamina $C$ e elevar a vitamina $C$ plasmática. Além disso, níveis plasmáticos significativamente maiores $(p<0,05)$ de GSH e ácido úrico foram encontrados nas fases suplemento e controle, respectivamente. A vitamina C oferecida tanto pela dieta como pelo uso de suplemento diminuiu os níveis plasmáticos de AST comparando-se ao controle. Contudo, nenhuma mudança foi encontrada nos valores plasmáticos de TBARS, hidroperóxido lipídico e CK após as diferentes formas de consumo de vitamina $C$.

O teste de correlação de Spearman revelou que quanto maior o consumo de AA exógeno, maiores os níveis de vitamina $C$ sérica antes do exercício $(r=0,62)$. O consumo de AA ainda apresenta correlação negativa com os níveis de TGO séricos antes do exercício $(r=0,61)$, logo após $(r=0,74)$ e 24 horas depois $(r=0,75)$, e com os níveis de ácido úrico, antes do exercício $(r=0,87)$, logo após $(r=0,70)$ e 24 horas depois $(r=0,71)$. A vitamina $C$ sérica apresentou correlação positiva com os níveis séricos de vitamina E 24 horas após o exercício na fase $\mathrm{S}(\mathrm{r}=0,69)$.

\section{DISCUSSÃO}

No presente estudo, o consumo adicional de AA, pela dieta ou pelo suplemento, propiciou uma melhora do sistema antioxidante e inibição de alguns biomarcadores de estresse oxidativo. O consumo de antioxidantes exógenos, através da alimentação, auxilia na capacidade antioxidante do organismo e alterações do status antioxidante podem ser relacionadas a um consumo alimentar inadequado e desbalanceado. No presente estudo, o único antioxidante da dieta que apresentou flutuação entre as fases - e por isso deve ser o responsável pelos resultados obtidos neste estudo - foi o AA (vitamina C). O uso de maiores quantidades de AA exógeno refletiu em uma maior concentração sérica deste antioxidante em relação à fase $C$. A maioria dos estudos feitos com atletas, que utilizaram suplemento de AA, obteve os mesmos resultados ${ }^{19,20}$. A resposta à suplementação pode ser determinada pelo conteúdo de vitamina $C$ nos suplementos, o consumo dietético e a concentração de AA no plasma antes da suplementação. Atletas com baixos níveis de AA circulantes têm uma melhor resposta à suplementação ${ }^{21}$.

Maiores quantidades de vitamina C sérica, no presente estudo, foram capazes de suprimir a liberação do antioxidante ácido úrico e inibir o aumento da lesão hepática e tecidual, medida pela AST, com o exercício e mesmo em repouso. Concordando com os achados de

Tabela 1. Valores médios do consumo habitual e do dia da coleta de antioxidantes nas diferentes fases do estudo.

\begin{tabular}{c|c|c|c|c|c|c|c}
\hline \multirow{2}{*}{ Nutrientes } & \multicolumn{2}{|c|}{ Fase controle (C) } & \multicolumn{2}{|c|}{ Fase dieta (D) } & \multicolumn{2}{c}{ Fase suplemento (S) } \\
\cline { 2 - 7 } & Consumo habitual & Dia da coleta & Consumo habitual & Dia da coleta & Consumo habitual & Dia da coleta \\
\hline Ácido ascórbico (mg) \# & $143,1 \pm 73,8^{\text {a }}$ & $171,9 \pm 150,4$ & $490,0 \pm 45,0 * \mathrm{~b}$ & $137,2 \pm 152,3 * *$ & $634,4 \pm 118,4^{* c}$ & $156,0 \pm 117,6^{* *}$ & $75-90 \mathrm{mg} / \mathrm{d}^{1}$ \\
\hline Vitamina E (mg) & $3,9 \pm 3,0$ & $4,5 \pm 4,3$ & $3,2 \pm 1,8$ & $3,3 \pm 2,8$ & $4,1 \pm 2,3$ & $3,4 \pm 2,5$ & $15 \mathrm{mg} / \mathrm{d}^{1}$ \\
\hline Retinol (Eq) & $699,4 \pm 195,9$ & $512,3 \pm 267,6$ & $759,5 \pm 333,2$ & $788,5 \pm 579,5$ & $807,8 \pm 220,5$ & $899,9 \pm 1075,4$ & $900 \mathrm{Eq} / \mathrm{d}^{1}$ \\
\hline Selênio (mg) & $84,7 \pm 62,0$ & $61,3 \pm 35,2$ & $70,3 \pm 34,2$ & $70,3 \pm 38,5$ & $79,3 \pm 23,6$ & $66,9 \pm 30,1$ & $55 \mathrm{mg} / \mathrm{d}^{1}$ \\
\hline Zinco (mg) & $8,6 \pm 3,5$ & $8,1 \pm 3,6$ & $10,6 \pm 4,1$ & $7,8 \pm 3,8$ & $11,5 \pm 5,2$ & $8,6 \pm 4,1$ & $11 \mathrm{mg} / \mathrm{d}^{1}$ \\
\hline a-tocoferol (mg) & $1,2 \pm 1,1$ & $1,1 \pm 1,1$ & $1,4 \pm 1,2$ & $1,4 \pm 1,8$ & $2,0 \pm 2,0$ & $1,4 \pm 1,2$ & 2 \\
\hline
\end{tabular}

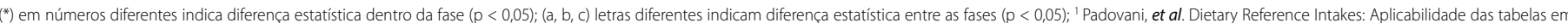
estudos nutricionais, 2006. ${ }^{2}$ Não possui valores de referência. \# Ácido ascórbico na segundo e terceira fases não representam o consumo habitual, pois possuem a adição de vitamina C pelo estudo. 


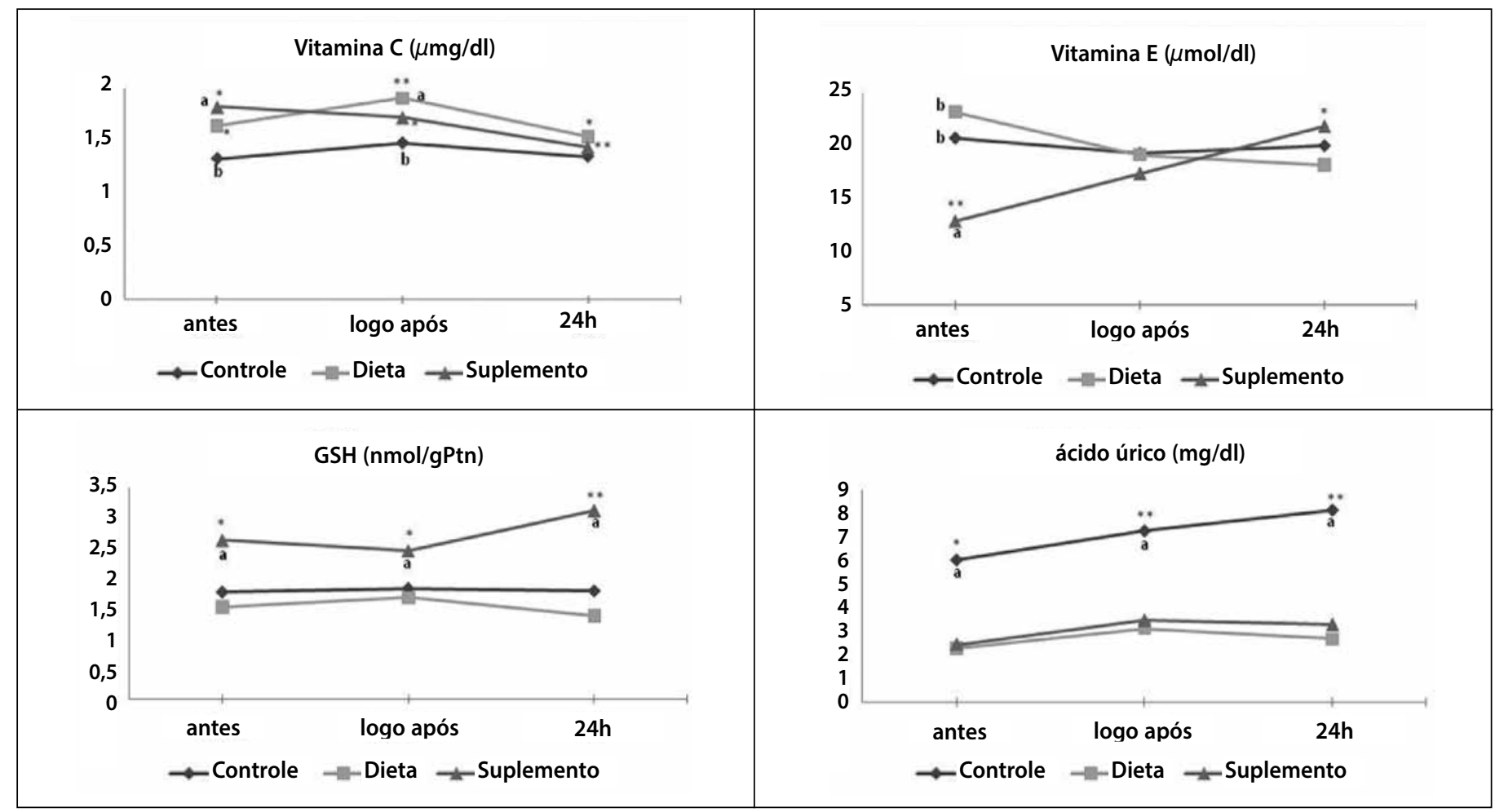

Figura 2. Concentrações plasmáticas médias dos antioxidantes vitamina C, vitamina E, glutationa (GSH) e ácido úrico nas diferentes fases: antes do exercício, logo após o exercício e 24 horas depois. $\left(^{*}\right)$ em números diferentes indica diferença estatística dentro da fase $(p<0,05)$. (a, b) letras diferentes indicam diferença estatística entre as fases $(p<0,05)$.

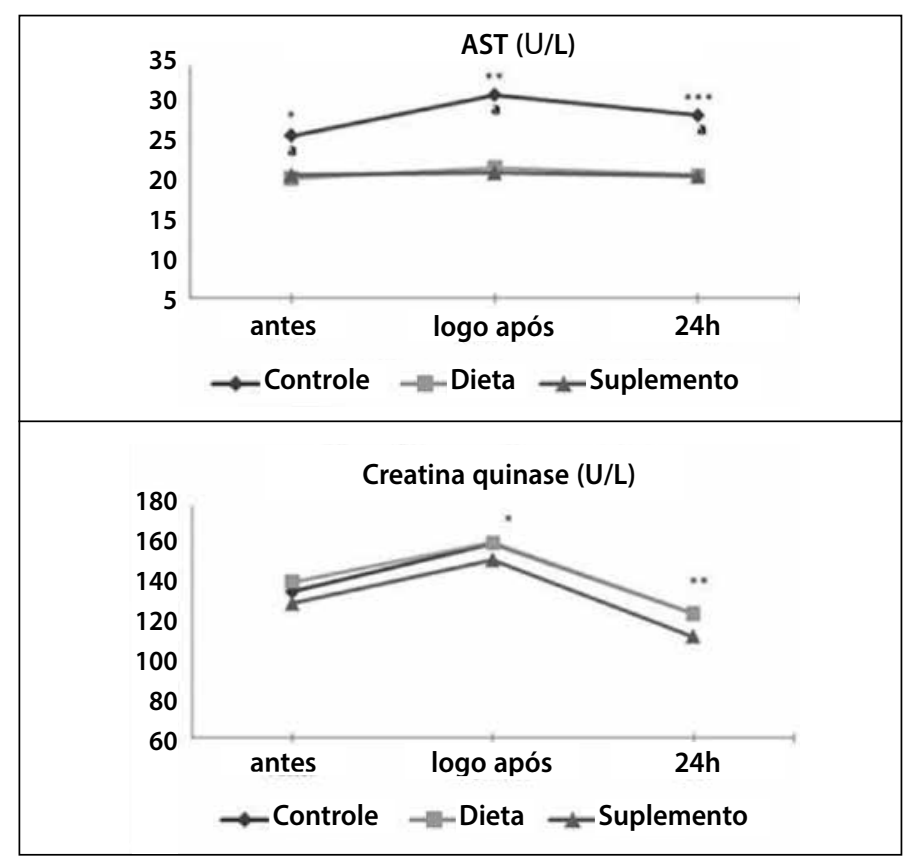

Figura 3. Concentrações plasmáticas médias dos marcadores de lesão hepática (aspartato aminotransferase - AST) e lesão muscular (creatina quinase - CK) nas diferentes

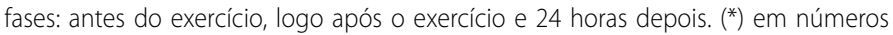
diferentes indica diferença dentro da fase $(p<0,05)$. $(a, b)$ letras diferentes indicam diferença entre as fases $(p<0,05)$.

Tauler et al. ${ }^{20}$ e Yanai e Marimoto ${ }^{22}$, em que o uso de suplemento de AA conteve a elevação de ácido úrico em atletas participantes de competição de duatlon e atletas bem treinados, respectivamente.

Ao mesmo tempo, na fase $C$, quando os níveis de vitamina $C$ sérica eram menores, o aumento de ácido úrico foi capaz de conter a peroxidação lipídica, já que não houve aumento dos níveis de TBARS. Sendo assim, o aumento de ácido úrico parece depender dos níveis plasmáticos de AA antes do exercício, já que ambos são fortes determinantes da capacidade antioxidante plasmática. Como descrito

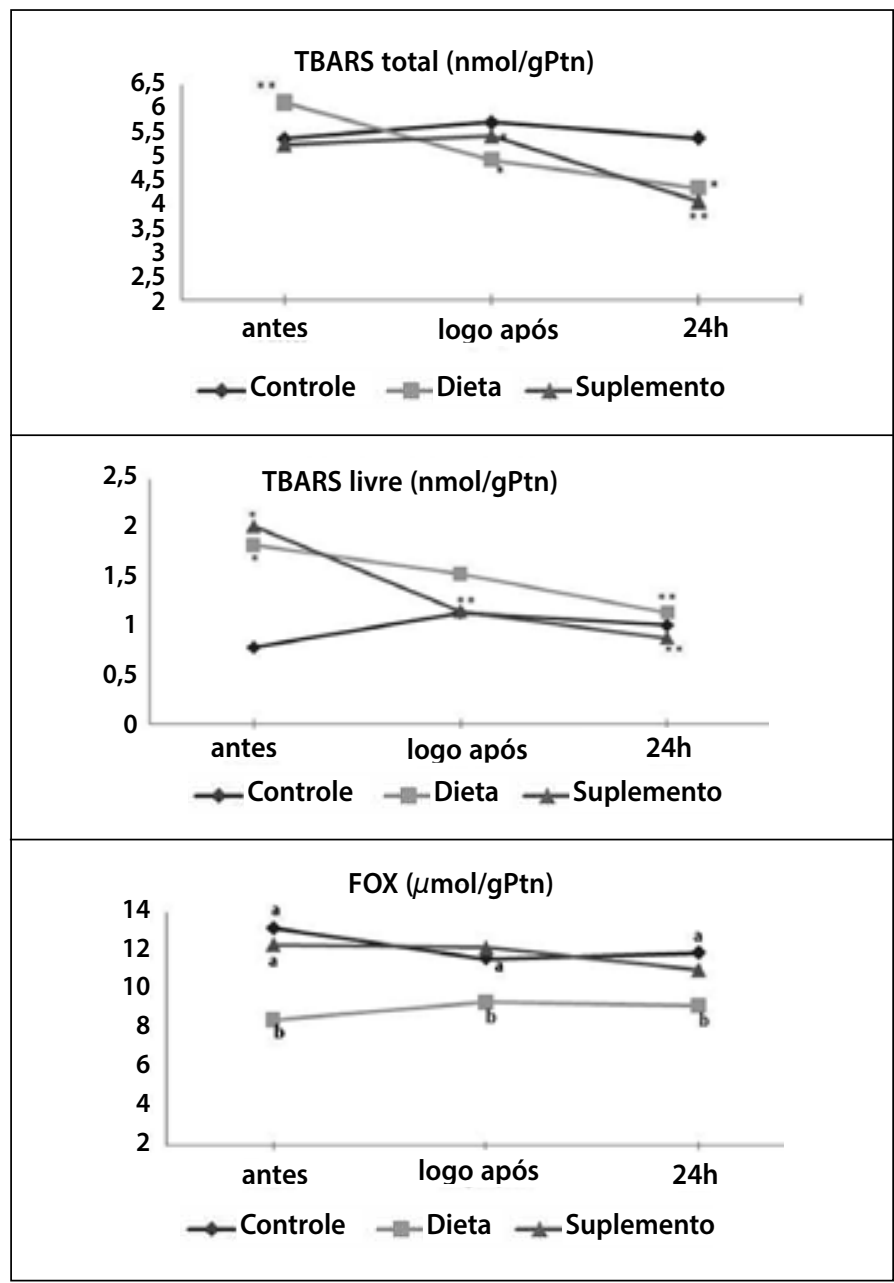

Figura 4. Concentrações plasmáticas médias dos marcadores de peroxidação lipídica TBARS total, TBARS livre e FOX nas diferentes fases: antes do exercício, logo após o exercício e 24 horas depois. (*) em números diferentes indica diferença dentro da fase $(P<0,05)$. (a, b) letras diferentes indicam diferença entre as fases $(p<0,05)$. 
por Sevanian et al.23, o ácido úrico pode substituir ou complementar algumas das funções do AA em humanos, poupando-o. Estudo feito com corredores ${ }^{24}$, competidores de meio ironman ${ }^{25}$ e atletas de rúgbi ${ }^{26}$ mostraram aumento de ácido úrico após treino intenso e competição, sugerindo que este aumento possa estar compensando a depleção de outros antioxidantes, por isso deve ser interpretado como um indicador de efeito cumulativo de treino intenso e competição.

Alguns estudos também concordam com os achados de relação inversa entre o consumo de antioxidante e o AST, marcador indireto de estresse oxidativo. Em corredores que não fizeram uso de suplemento antioxidante, houve aumento de AST após corrida de 50 e $80 \mathrm{~km}^{24}$. Da mesma maneira, estudo feito com camundongos, sob treinamento de natação e sem suplementação antioxidante, mostrou que o processo oxidativo gerado pelo exercício físico contribui com eventos de lesão celular, observados pelo aumento nos valores de CK e AST ${ }^{27}$. Sendo assim, a fase em que os indivíduos não receberam adição de AA à dieta apresentou maior nível de estresse oxidativo, devido aos maiores valores séricos de AST que as outras fases.

Diferente dos marcadores de estresse oxidativo discutidos acima, a peroxidação lipídica, analisada pelo TBARS total e livre, não foi diferente entre as fases em função do exercício. Apesar disso, na fase $C$ tanto o TBARS total como o livre se comportou de maneira constante, enquanto nas fases D e $S$ houve diminuição dos níveis após o exercício, ou seja, maiores níveis de vitamina $C$ séricos nestas duas fases foram suficientes para conter a peroxidação. Enquanto FOX, outro marcador de peroxidação lipídica com ação nos hidroperóxidos, foi menor com o uso de dieta rica em $A A$, sugerindo que dieta rica em $A A$ permite tirar vantagem em relação ao uso de um suplemento de AA, pelo efeito aditivo e sinérgico dos fenólicos que favorecem uma maior concentração de antioxidantes ${ }^{28,29}$.

Em relação aos níveis séricos de antioxidantes, apenas a vitamina C apresentou variação com o exercício, enquanto as concentrações de vitamina E e GSH não modificaram em decorrência do mesmo. A redução dos valores de AA algumas horas após o exercício pode sugerir alterações na demanda antioxidante dos tecidos ${ }^{19}$. Sendo assim, no presente estudo, a redução nos níveis 24 horas após o exercício nas fases $D$ e $S$ pode estar relacionada com o mecanismo homeostático que regula os níveis de $A A$ no plasma. Já, na fase $C$, os níveis de $A A$ não variaram com o exercício, o que pode ter sido consequência do grande aumento de ácido úrico poupando o uso de vitamina C, como descrito anteriormente.

As divergências entre os estudos sobre estresse oxidativo são, provavelmente, devidas às diferenças metodológicas, tipo, intensidade e duração do exercício ${ }^{30}$, nível de aptidão dos sujeitos, alimentação ${ }^{24}$, idade e sexo ${ }^{31}$, escolha do biomarcador, tipo de amostra, quantidade e duração do tratamento antioxidante ${ }^{3}$. Portanto, é recomendado que futuras investigações empreguem protocolos de exercício suficientes, utilizem uma completa avaliação de biomarcadores de estresse oxidativo e façam múltiplas amostras após o exercício na tentativa de oferecer achados válidos e significativos ${ }^{3}$.

\section{CONCLUSÃO}

O uso de dieta rica em AA e suplementação de AA favoreceram uma menor peroxidação lipídica em nadadores de elite e diminuição da peroxidação lipídica após exercício. Sem uso de nenhuma adição de AA houve um maior dano hepático e tecidual, menores níveis de antioxidantes (vitamina C e GSH) e o aumento de ácido úrico, que aumenta quando há grande quantidade de radicais livres presentes, e que protegeu os atletas da peroxidação lipídica quando eles possuíam menor poder de outros antioxidantes.

Todos os autores declararam não haver qualquer potencial conflito de interesses referente a este artigo.

\section{REFERÊNCIAS}

1. Cruzat VF, Rogero MM, Borges MC, Tirapegui J. Current aspects about oxidative stress, physical exercise and supplementation. Rev Bras Med Esporte 2007;13:304-10.

2. Michailidis $Y$, Jamurtas AZ, Nikolaidis MG, Fatouros IG, Koutedakis Y, Papassotiriou I, Kouretas D. Sampling time is crucial for measurement of aerobic exercise-induced oxidative stress. Med Sci Sports Exerc 2007;39:1107-13.

3. Fisher-Wellman K, Bloomer RJ. Acute exercise and oxidative stress: a 30 year history. Dyn Med 2009;13;8:1

4. Bloomer RJ, Goldfarb AH, McKenzie MJ. Oxidative stress response to aerobic exercise: comparison of antioxidant supplements. Med Sci Sports Exerc 2006;38:1098-105.

5. Gougoura S, Nikolaidis MG, Kostaropoulos IA, Jamurtas AZ, Koukoulis G, Kouretas D. Increased oxidative stress indices in the blood of child swimmers. Eur J Appl Physiol 2007;100:235-9.

6. Goldfarb AH, Garten RS, Cho C, Chee PD, Chambers LA. Effects of a fruit/berry/vegetable supplement on muscle function and oxidative stress. Med Sci Sports Exerc 2011;43:501-8.

7. Nakhostin-Roohi B, Babaei P, Rahmani-Nia F, Bohlooli S. Effect of vitamin C supplementation on lipid peroxidation, muscle damage and inflammation after 30-min exercise at 75\% $\mathrm{VO}_{2 \max }$.J Sports Med Phys Fitness 2008;48:217-24.

8. Rodriguez NR, DiMarco NM, Langley S; American Dietetic Association; Dietitians of Canada; American College of Sports Medicine. Nutrition and Athletic Performance. J Am Diet Assoc. 2009;109:509-27.

9. Panza VP, Coelho MSPH, Pietro PFD, Assis MAA, Vasconcelos FAG. Consumo alimentar de atletas: reflexões sobre recomendações nutricionais, hábitos alimentares e métodos para avaliação do gasto e consumo energéticos. Rev Nutr 2007;20:681-92

10. Finaud J, Lac G, Filaire E. Oxidative stress: relationship with exercise and training. Sports Med 2006;36:327-58.

11. Margaritis I, Palazzetti S, Rousseau AS, Richard MJ, Favier A. Antioxidant supplementation and tapering exercise improve exercise-induced antioxidant response. J Am Coll Nutr 2003;22:147-56.

12. Padovani RM, Amaya-Farfán J, Colugnati FAB, Domene SMA. Dietary reference intakes: Aplicabilidade das TABLES em estudos nutricionais. Rev Nutr 2006;19:741-60.

13. Cuppari L, Schor N. Guia de Nutrição: nutrição clínica do adulto. $2^{\mathrm{a}}$ ed., Barueri: Manole, 2005.

14. Buege JA, Aust SD. Microsomal lipid peroxidation. Meth Enzymology 1978:52:302-10.

15. Södergren E, Nourooz-Zadeh J, Berglund L, Vessby B. Re-evaluation of the ferrous oxidation in xylenol orange assay for the measurement of plasma lipid hydroperoxides. J Biochem Biophys Methods 1998;37:137-46.

16. Sedlak J, Lindsay RH. Estimation of total, protein-bound, and nonprotein sulfhydryl groups in tissue with Ellman's reagent. Analytical Biochem 1968;25:192-205.

17. Bessey OA. Ascorbic acid microchemical methods. In: Vitamin Methods. New York: Academic Press, 1, 303, 1960.
18. Jordão AA Jr, Chiarello PG, Arantes MR, Meirelles MS, Vannucchi H. Effect of an acute dose of ethanol on lipid peroxidation in rats: action of vitamin E. Food Chem Toxicol 2004;42:459-64.

19. Peake JM. Vitamin C: effects of exercise and requirements with training. Int J Sport Nutr Exerc Metab 2003;13:125-51.

20. Tauler P, Aguiló A, Gimeno I, Fuentespina E, Tur JA, Pons A. Influence of vitamin C diet supplementation on endogenous antioxidant defences during exhaustive exercise. Pflugers Arch 2003;446:658-64.

21. Naziroğlu M, Kilinç F, Uğuz AC, Celik O, Bal R, Butterworth PJ, Baydar ML. Oral vitamin C and E combination modulates blood lipid peroxidation and antioxidant vitamin levels in maximal exercising basketball players. Cell Biochem Funct 2010;28:300-5.

22. Yanai $\mathrm{H}$, Morimoto $\mathrm{M}$. Effect of ascorbate on serum lipids and urate metabolism during exhaustive training. Clin Sci (Lond) 2004;106:107-9.

23. Sevanian A, Davies KJ, Hochstein P. Serum urate as an antioxidant for ascorbic acid. Am J Clin Nutr 1991;54(6 Suppl):1129S-34S.

24. Chevion S, Moran DS, Heled Y, Shani Y, Regev G, Abbou B, Berenshtein E, Stadtman ER, Epstein Y. Plasma antioxidant status and cell injury after severe physical exercise. Proc Natl Acad Sci U S A 2003 29;100:5119-23.

25. Schneider CD, Silveira MM, Moreira JCF, Belló-Klein A, Oliveira AR. Efeito do Exercício de Ultrarresistência Sobre Parâmetros de Estresse Oxidativo. Rev Bras Med Esporte 2009:15:89-92.

26. Finaud J, Scislowski V, Lac G, Durand D, Vidalin H, Robert A, Filaire E. Antioxidant status and oxidative stress in professional rugby players: evolution throughout a season. Int J Sports Med 2006;27:87-93.

27. Antunes Neto JMF, Rivera RJB, Calvi RG, Raffa MF, Donadon CC, Pereira AG. Níveis comparativos de estresse oxidativo em Camundongos em Duas Situações do Limite Orgânico: Overreaching Induzido por Treinamento de Natação e Câncer. Rev Bras Med Esporte 2008;14:548-52

28. Guimarães R, Barros L, Barreira JC, Sousa MJ, Carvalho AM, Ferreira IC. Targeting excessive free radicals with peels and juices of citrus fruits: grapefruit, lemon, lime and orange. Food Chem Toxicol 2010;48:99-106.

29. Vanamala J, Reddivari L, Yoo KS, Pike, LM, Patil BS. Variation in the content of bioactive flavonoids in different brands of orange and grapefruit juices. J Food Comp Analysis 2006;19:157-66.

30. Goldfarb AH, Patrick SW, Bryer S, You T. Vitamin C supplementation affects oxidative-stress blood markers in response to a 30-minute run at 75\% $\mathrm{VO}_{2 \text { max }}$ Int J Sport Nutr Exerc Metab 2005;15:279-90.

31. Cooper CE, Vollaard NB, Choueiri T, Wilson MT. Exercise, free radicals and oxidative stress. Biochem Soc Trans 2002:30:280-5 